\title{
The Optimal Screw Length of Lumbar Pedicle Screws during Minimally Invasive Surgery Fixation: A Computed Tomography-Guided Evaluation of 771 Screws
}

\author{
Min Jia Chua ${ }^{1}$, Shiblee Siddiqui ${ }^{2}$, Chun Sing Yu ${ }^{1}$, Colum Patrick Nolan ${ }^{3}$, Jacob Yoong-Leong $\mathrm{Oh}^{1}$ \\ ${ }^{I}$ Department of Orthopaedic Surgery, Tan Tock Seng Hospital, Singapore \\ ${ }^{2}$ Department of Orthopedics, Wockhardt Hospital, Mumbai, India \\ ${ }^{3}$ Department of Neurosurgery, National Neuroscience Institute, Tan Tock Seng Hospital, Singapore
}

\begin{abstract}
Study Design: A retrospective study of radiographic parameters of patients who underwent lumbar spinal pedicle screw insertion. Purpose: The optimal length of pedicle screws is often determined by the lateral radiograph during minimally invasive surgery (MIS). Compared with open techniques, measuring the precise length of screws or assessing the cortical breach is challenging. This study aims to ascertain the optimal pedicle screw lengths on intraoperative lateral radiographs for L1-L5.

Overview of Literature: Research has revealed that optimal pedicle screw length is essential to optimize fixation, especially in osteoporotic patients; however, it must be balanced against unintentional breach of the anterior cortex, risking injury to adjacent neurovascular structures as demonstrated by case reports.

Methods: We reviewed intra- and postoperative computed tomography scans of 225 patients who underwent lumbar pedicle screw insertion to ascertain which of the inserted screws were 'optimal screws.' The corresponding lengths of these screws were analyzed on postoperative lateral radiographs to ascertain the ideal position that a screw should attain (expressed as a percentage of the entire vertebral body length).

Results: We reviewed 880 screws of which 771 were optimal screws. We noted a decreasing trend in average optimal percentages of insertion into the vertebral body for pedicle screws going from L1 (average $=87.60 \%$ ) to $L 5$ (average $=78.87 \%$ ). The subgroup analysis revealed that there was an increasing percentage of screws directed in a straight trajectory from $L 1$ to $L 5$, compared to a medially directed trajectory.

Conclusions: During MIS pedicle screw fixation, this study recommends that pedicle screws should not exceed $85 \%$ of the vertebral body length on the lateral view for $\mathrm{L} 1,80 \%$ for $\mathrm{L} 2-\mathrm{L} 4$, and $75 \%$ for $\mathrm{L} 5$; this will minimize the risk of anterior cortical breach yet maximize pedicle screw purchase for fixation stability.
\end{abstract}

Keywords: Lumbar vertebrae; Pedicle screws; Minimally invasive surgery; Retrospective studies; Radiography

Received Oct 23, 2018; Revised Jan 22, 2019; Accepted Mar 1, 2019

Corresponding author: Jacob Yoong-Leong Oh

Department of Orthopaedic Surgery, Tan Tock Seng Hospital, 11 Jalan Tan Tock Seng, Singapore 308433

Tel: +65-91555918, Fax:+65-63577715, E-mail: Jacob_YL_OH@ttsh.com.sg 


\section{Introduction}

Pedicle screw fixation is the gold standard for internal fixation of the thoracic and lumbar spine [1,2]. Contrary to open surgical techniques where using instruments, such as the ball-tip pedicle probe, enable surgeons to validate the length of the screw and determine cortical breaches, it is not feasible in the minimally invasive surgery (MIS) approach. Surgeons using the MIS technique often depend on other surrogate measures, such as lateral radiographs and preoperative imaging modalities (computed tomography $[\mathrm{CT}]$ and magnetic resonance imaging scans), to ascertain the optimal pedicle screw insertion within the vertebral body.

This study aims to ascertain the optimal pedicle screw insertion lengths on intraoperative lateral radiographs during MIS surgery for different lumbar levels from L1 to L5 to attain maximal length safely without causing any anterior cortical breach.

\section{Materials and Methods}

This is a retrospective study of radiographic parameters of all patients who underwent spinal surgeries involving lumbar pedicle screw insertion at our institution. We reviewed intraoperative CT scans (for patients who had pedicle screw insertion under O-arm navigation) or postoperative CT scans (for patients who underwent pedicle screw insertion under II guidance but who had postoperative CT scans performed for either their spinal condition or other dissimilar conditions, including CT of the abdomen and pelvis for general surgical indications) to define optimal screws. Of note, we excluded patients who did not have any CT scans performed enabling assessment.

In this study, we defined an 'optimal screw' as one that was within $5 \mathrm{~mm}$ of the anterior cortex of the vertebral body and did not have any anterior cortical breach (Fig. 1); this was defined retrospectively from the CT scans. Furthermore, this was considered an 'optimal' screw in this study because it was the longest possible screw that did not cause a breach, enabling the best purchase possible.

After determining the optimal screws, we reviewed lateral radiographs of the spine to evaluate the length of these selected screws in the vertebral body on X-rays (Fig. 2). After that, we evaluated the percentage of the length of the screws in individual vertebral bodies, providing the optimal screw length percentages at each lumbar vertebral level for respective patients. As part of our subgroup analysis, screws were also classified into medially or straight directed based on the trajectory and the location of the tip of the screw in the vertebral body on the axial and coronal views of the CT scan (Fig. 3).

This study was exempted from institutional review board as it was a retrospective study using radiographic imaging parameters without any identifiable data for the patients.

\section{Results}

Of 517 patients who underwent lumbar instrumentation,

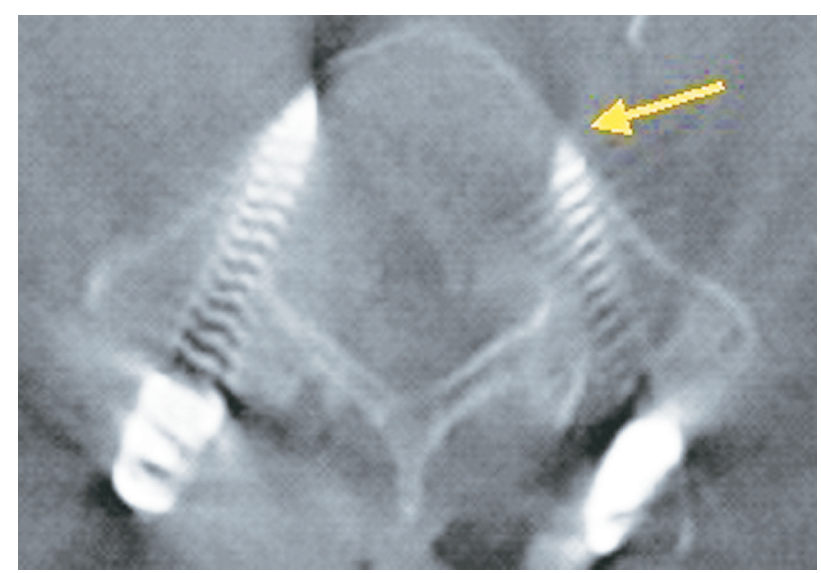

Fig. 1. The axial computed tomography image with an arrow pointing to 'optimal screw' with no breach and within $5 \mathrm{~mm}$ of the anterior cortex of the vertebral body.

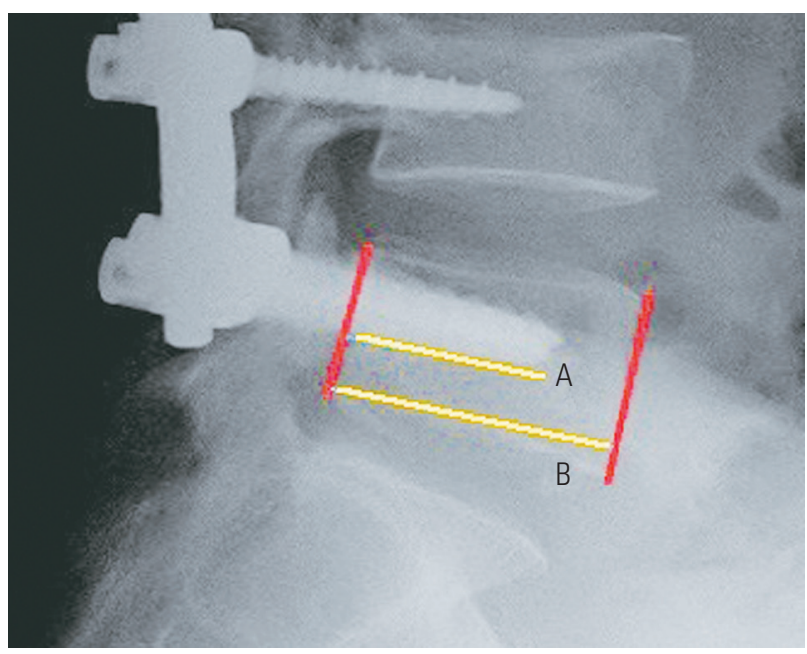

Fig. 2. The lateral spinal radiograph showing how measurements were performed ( $\mathrm{A} / \mathrm{B}$ with $\mathrm{A}$ being screw length insertion and $\mathrm{B}$ being vertebral body length). 

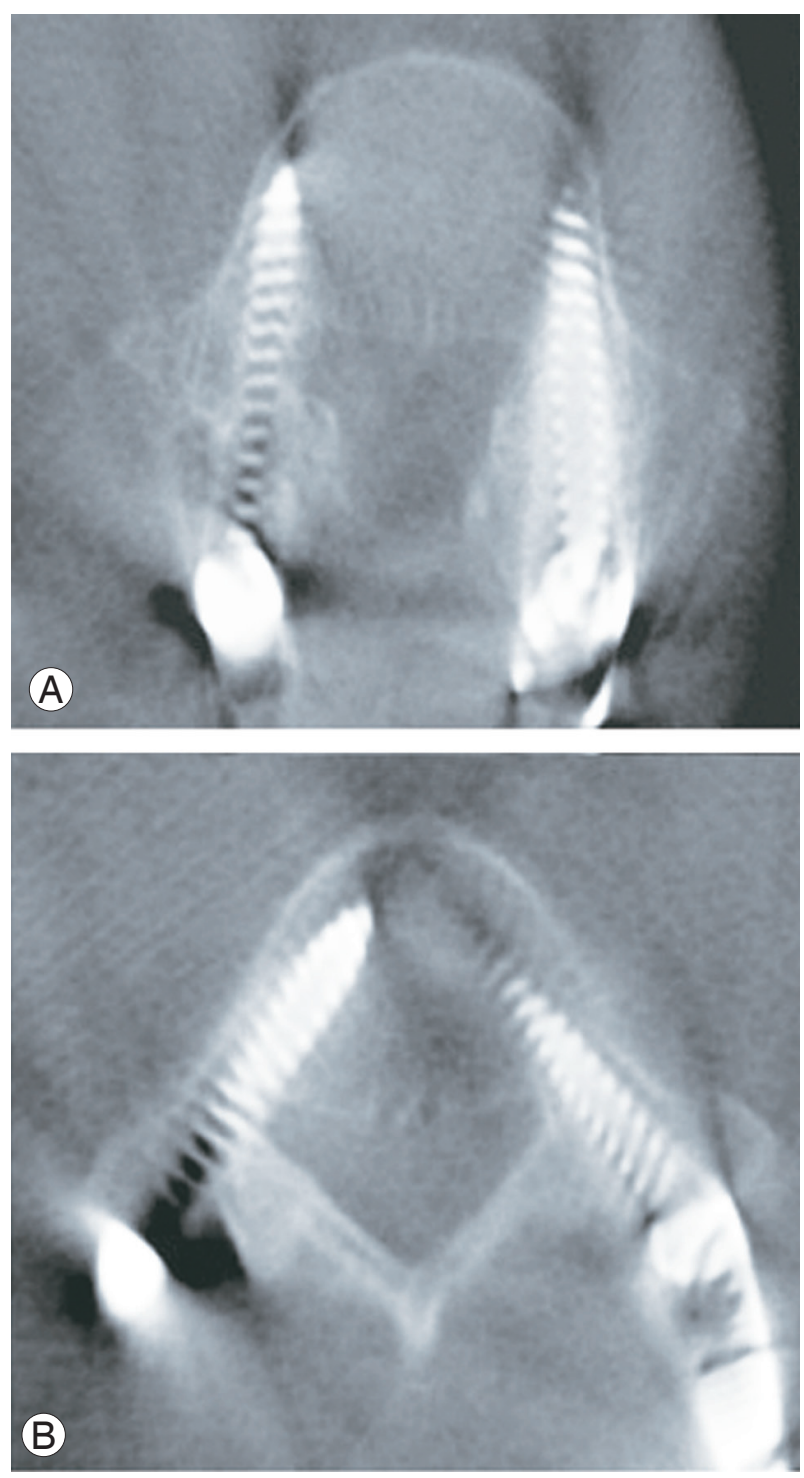

Fig. 3. The computed tomography scan showing the straight (A) and medially directed (B) screws in the axial view.

we enrolled 225 based on our inclusion criteria; of these, $\mathrm{O}$-arm navigation was used for surgery in 167 patients (74.2\%), whereas II was used in 58 patients $(25.8 \%)$. Of these 225 patients, we analyzed 881 pedicle screws; of these, 770 were optimal screws based on the previously mentioned criteria and were, thus, included in the study. Table 1 summarizes further breakdown of the screws in this study.

After selecting the optimal screws and reviewing radiographs, we calculated the average percentages of the insertion of the pedicle screw into the vertebral body. The average percentages of the optimal length of the pedicle screws at various levels were as follows (with 95\% con-
Table 1. Numerical breakdown of the pedicle screws by lumbar spinal level

\begin{tabular}{lc} 
Vertebral body level & Total no. of screws \\
L1 & 29 \\
L2 & 51 \\
L3 & 110 \\
L4 & 264 \\
L5 & 316 \\
\hline
\end{tabular}

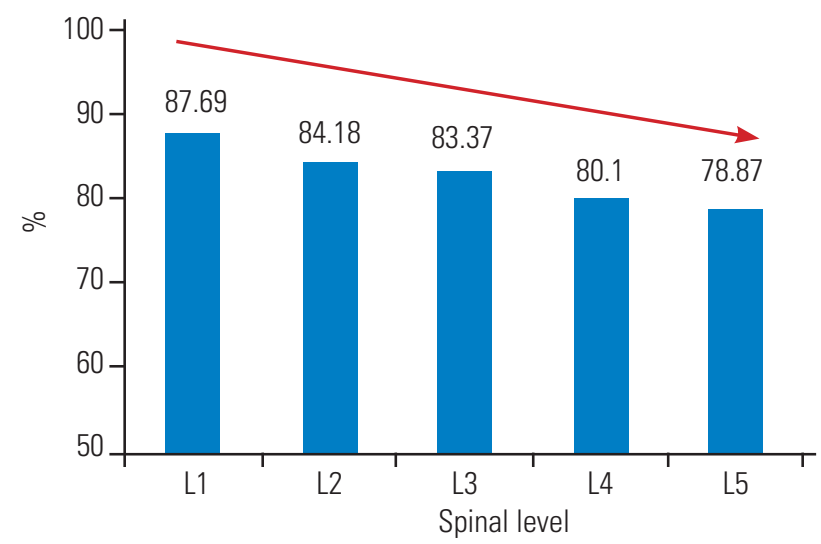

Fig. 4. The graphical depiction of the average percentage of the optimal screw insertion into the vertebral body from $L 1$ to $L 5$.

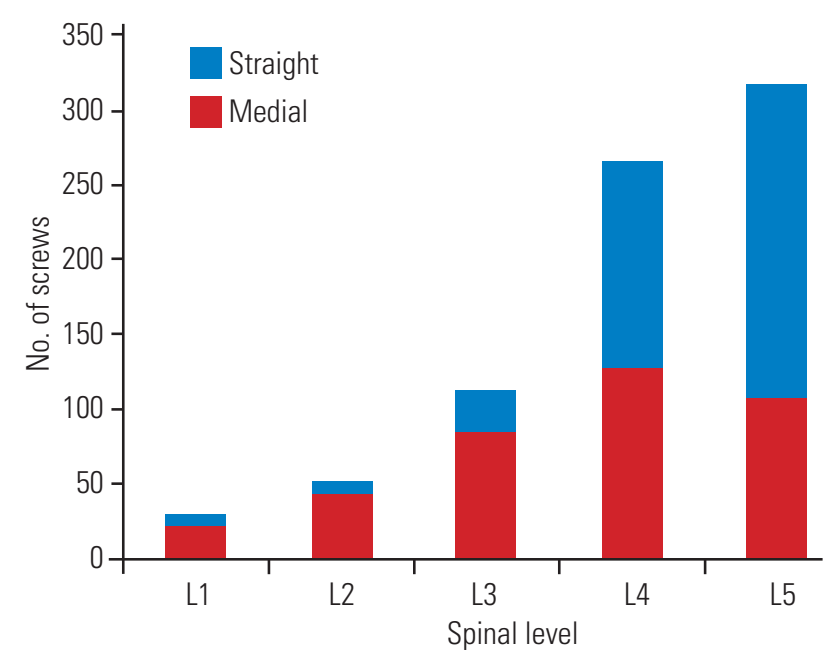

Fig. 5. A chart showing the breakdown of screws directed in a straight and medial trajectory at the different lumbar levels.

fidence intervals): $87.69 \% \pm 2.67 \%$ for L1; $84.18 \% \pm 1.85 \%$ for L2; $83.37 \% \pm 1.34 \%$ for $\mathrm{L} 3$; $80.10 \% \pm 0.96 \%$ for L4; and $78.87 \% \pm 0.91 \%$ for L5. We observed a decreasing trend from L1 to L5 (Fig. 4).

In addition, the subgroup analysis revealed an increasing percentage of screws that were directed in a straight 
Table 2. Average percentage of insertion of medially directed and straight screws from L1 to $L 5$ with $p$-value

\begin{tabular}{lccc} 
Vertebral body level & Medial screws & Straight screws & $p$-value \\
L1 & $89.79 \pm 2.78$ & $82.19 \pm 4.76$ & 0.01208 \\
L2 & $85.40 \pm 1.96$ & $78.50 \pm 3.17$ & 0.004131 \\
L3 & $84.44 \pm 1.45$ & $79.90 \pm 2.86$ & 0.004334 \\
L4 & $83.85 \pm 1.09$ & $76.63 \pm 1.30$ & $<0.00001$ \\
L5 & $82.61 \pm 1.38$ & $77.02 \pm 1.10$ & $<0.00001$ \\
\hline
\end{tabular}

Values are presented as average $\% \pm 95 \%$ confidence intervals.
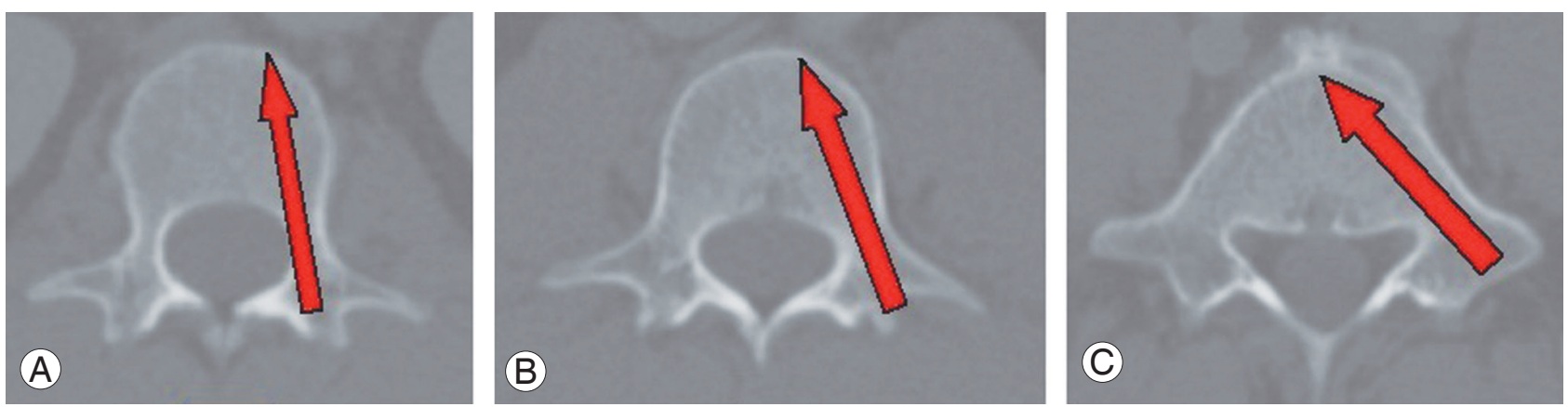

Fig. 6. Axial computed tomography images of a patient's L1 (A), L3 (B), and L5 (C) vertebral bodies showing the morphology and increasing medial trajectories of screws needed to achieve optimal pedicle screw fixation (arrow).

trajectory from L1 to L5 (Fig. 5). For L5, nearly two-thirds of the screws were inserted in a straight trajectory, possibly depicting the challenges in medializing the screw trajectory because of anatomical variances. Furthermore, the statistical analysis revealed that medially directed screws were significantly longer $(p<0.05)$ than screws inserted into a straight trajectory, with differences in the average percentage of screws in the vertebral body ranging from $4.54 \%$ to $7.60 \%$ (Table 2 ).

\section{Discussion}

Pedicle screw fixation of the lumbar spine is a frequently used procedure in spinal surgery in combination with arthrodesis to attain stabilization [3]. It is imperative to attain the optimal pedicle screw length to optimize fixation $[4,5]$; this is particularly crucial in an aging population of osteoporotic patients [6].

The vertebral body takes on a more oval-shaped morphology going down the lumbar spinal levels from L1 to L5, and medial angulation of the screws is needed to optimize the length of the pedicle screw length inserted into the vertebral body (Fig. 6); however, this might not always be feasible because of anatomical variants, like a high

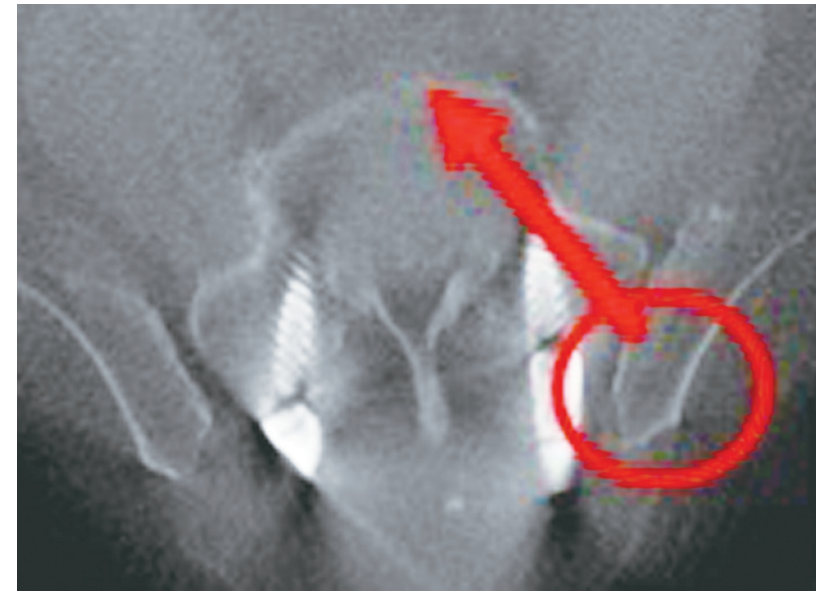

Fig. 7. Axial computed tomography image of a patient with high iliac crest precluding lateral pedicle screw entry point for medial screw angulation (arrow). Hence, a straightforward trajectory has been used.

iliac crest, which might preclude a lateral pedicle screw entry point essential for medial angulation of the screw (Fig. 7) as well as soft tissue interference in obese patients. Thus, a straight trajectory might need to be utilized for pedicle screw insertion, especially at L5. This variation in screw trajectory might mislead surgeons into inadvertently breaching the anterior cortex of the vertebral body 

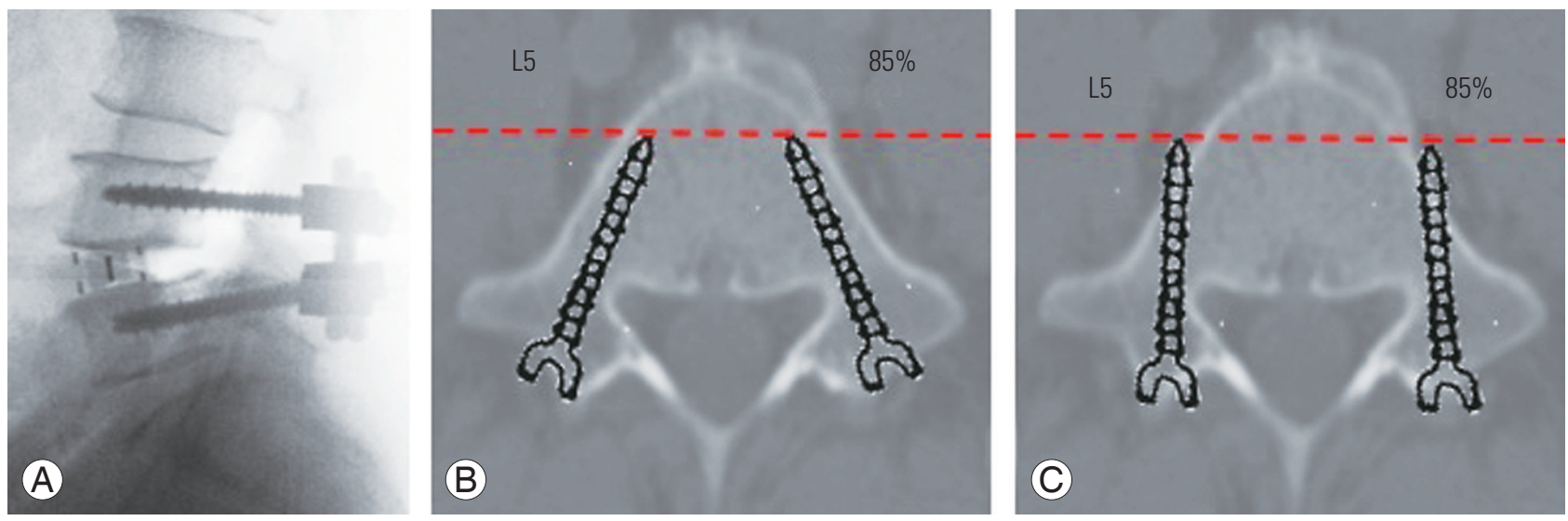

Fig. 8. The lateral radiograph of pedicle screws inserted at $L 4$ and $L 5(A)$, axial computed tomography image of $L 5$ vertebral body with schematic drawing showing optimal pedicle screw insertion in a medialized trajectory to $85 \%$ of the vertebral length (B), schematic drawing showing anterior cortical breach in a straight trajectory to $85 \%$ of the vertebral length (C).

if a straightforward trajectory is utilized. For the same length of screw insertion on a lateral image projection, it might, in fact, cause anterior cortical breach depending on whether or not the screw has been inserted in a medialized trajectory, thereby risking injury to the adjacent neurovascular structures (Fig. 8).

This study aimed to determine the optimal pedicle screw length at various lumbar levels by correlating with lateral radiographs of optimal screws that had been determined on CT images, thereby serving as a guide for MIS pedicle screw insertion. We found that the overall screw lengths on the lateral radiograph, which corresponded to an optimal screw length, ranged from $87.69 \%$ for L1 to $78.87 \%$ for L5, with a declining percentage going from L1 to L5. These percentages serve as a guide for surgeons who use intraoperative II to guide pedicle screw insertion under the MIS technique. Compared with open techniques, surgeons are not able to 'feel' for an anterior breach but have to depend entirely on lateral fluoroscopic images to guide their screw length. The percentages in this study, thus, guide surgeons to 'maximize' the screw length to get optimal purchase while having a good safety margin to minimize the risk of an anterior cortical breach and injury to adjacent neurovascular structures $[7,8]$.

Of note, overzealous insertion of the pedicle screw to a deeper depth could risk breach of the anterior cortex and exacerbate risks of injury to adjacent structures, whereas shorter screws might case insufficient vertebral body insertion, compromising the pullout strength of the screw and fixation. This is especially vital for L5, as the increased need for straight trajectories of screws because of ana- tomical variants means that there is an elevated risk of anterior cortical breach.

This study has some limitations. First, the number of pedicle screws for the upper lumbar levels of L1 and L2 was relatively low. In addition, we define an optimal screw with regard to the distance of the tip to the anterior cortex to optimize fixation; however, we did not perform biomechanical pullout strength testing in this study. Nevertheless, some studies have reported that enhancement in the strength of the transpedicular screw-vertebra interface could be attained by increasing the depth of screw insertion. For example, Krag et al. [9] reported that the mean failure strength of screws at $50 \%$ depth was $75 \%-77 \%$ of that at $80 \%$ depth, and the strength for screws at $100 \%$ ('to-cortex') depth was $124 \%-154 \%$ of that at $80 \%$. Furthermore, radiographic measurements in this study were performed by two observers, which could have introduced interobserver variability; this was, however, mitigated by corroborating the measurements between both observers, which exhibited good interobserver concordance and reliability.

\section{Conclusions}

This study recommends that during the MIS pedicle screw fixation, pedicle screws should not exceed $85 \%$ of the vertebral body length on the lateral view for L1, $80 \%$ of the vertebral body length for L2-L4, and 75\% of the vertebral body length for L5; this would help minimize the risk of anterior cortical breach yet maximize pedicle screw purchase for fixation stability. 


\section{Conflict of Interest}

No potential conflict of interest relevant to this article was reported.

\section{References}

1. Gaines RW Jr. The use of pedicle-screw internal fixation for the operative treatment of spinal disorders. J Bone Joint Surg Am 2000;82:1458-76.

2. Denis F. The three column spine and its significance in the classification of acute thoracolumbar spinal injuries. Spine (Phila Pa 1976) 1983;8:817-31.

3. Yahiro MA. Comprehensive literature review: pedicle screw fixation devices. Spine (Phila Pa 1976) 1994;19:2274S-8S.

4. Buckenmeyer LE. Optimization of pedicle screw depth in the lumbar spine: biomechanical characterization of screw stability and pullout strength [dissertation]. Toledo (OH): The University of Toledo; 2013.

5. Karami KJ, Buckenmeyer LE, Kiapour AM, et al.
Biomechanical evaluation of the pedicle screw insertion depth effect on screw stability under cyclic loading and subsequent pullout. J Spinal Disord Tech 2015;28:E133-9.

6. Battula S, Schoenfeld A, Vrabec G, Njus GO. Experimental evaluation of the holding power/stiffness of the self-tapping bone screws in normal and osteoporotic bone material. Clin Biomech (Bristol, Avon) 2006;21:533-7.

7. Watanabe K, Yamazaki A, Hirano T, et al. Descending aortic injury by a thoracic pedicle screw during posterior reconstructive surgery: a case report. Spine (Phila Pa 1976) 2010;35:E1064-8.

8. Foxx KC, Kwak RC, Latzman JM, Samadani U. A retrospective analysis of pedicle screws in contact with the great vessels. J Neurosurg Spine 2010;13:403-6.

9. Krag MH, Beynnon BD, Pope MH, DeCoster TA. Depth of insertion of transpedicular vertebral screws into human vertebrae: effect upon screw-vertebra interface strength. J Spinal Disord 1988;1:287-94. 